\title{
Cobertura profilática para lesões por pressão na face dos profissionais de saúde durante a COVID-19
}

RESUMO | Objetivo: propor a elaboração de cobertura profilática para prevenção de lesão por pressão na face de profissionais de saúde pelo uso de máscara durante a pandemia provocada pela Covid-19. Método: trata-se de um estudo metodológico dividido em 2 etapas: revisão narrativa de literatura e elaboração da cobertura profilática, utilizando critérios pré-estabelecidos pelos pesquisadores. Resultados: a cobertura profilática, proposta neste estudo, tem apresentação em rolo, não estéril e deverá ser utilizada somente em pele íntegra. Será composta por uma fita de silicone macio, perfurado, com micro aderência, de $2,5 \mathrm{~cm}$ de largura; camada central de espuma extrafina de poliuretano, com $1 \mathrm{~cm}$ de largura; e parte externa de filme de poliuretano. Conclusão: a proposta da cobertura profilática promoverá a absorção da umidade, reduzirá a pressão e o cisalhamento e, consequentemente, as lesões por pressão na face de profissionais de saúde pelo uso de máscaras de proteção individual.

Palavras-chaves: Prevenção e Controle; Curativos oclusivos; Úlcera por pressão; Equipamentos e Provisões.

ABSTRACT | Objective: To propose the development of prophylactic dressing to prevent pressure injuries on the face of health professionals by the use of a mask during the pandemic caused by Covid-19. Methods: This a methodological study divided into two stages: narrative review of the literature and elaboration of prophylactic dressing, using pre-established criteria by the researchers. Results: The prophylactic dressing, proposed in this study, has a roll presentation, not sterile and should be used only on intact skin. It will be composed of a soft silicone tape, perforated, with micro-adherence, $2.5 \mathrm{~cm}$ wide; central layer of extra-fine polyurethane foam, with $1 \mathrm{~cm}$ wide; and external polyurethane film. Conclusions: The prophylactic dressing proposal will promote moisture absorption, will reduce pressure and shear and, consequently, pressure injuries on the face of health professionals through the use of individual protection masks.

Keywords: Prevention \& Control; Occlusive dressings; Pressure ulcer; Equipment and Supplies.

RESUMEN | Objetivo: proponer el desarrollo de coberturas profilácticas para prevenir lesiones por presión en el rostro de los profesionales de la salud mediante el uso de mascarilla durante la pandemia causada por Covid-19. Métodos: se trata de un estudio metodológico dividido en 2 etapas: revisión narrativa de la literatura y elaboración de la cobertura profiláctica, utilizando criterios preestablecidos por los investigadores. Resultados: la cobertura profiláctica, propuesta en este estudio, tiene presentación en rollo, no es estéril y debe usarse solo sobre piel intacta. Consistirá en una cinta de silicona microadhesiva perforada, suave y de 2,5 cm de ancho; capa central de espuma de poliuretano extrafina de $1 \mathrm{~cm}$ de ancho; y parte exterior de película de poliuretano. Conclusión: la propuesta de cobertura profiláctica promoverá la absorción de humedad, reducirá la presión y el cizallamiento y, en consecuencia, las lesiones por presión en el rostro de los profesionales de la salud mediante el uso de máscaras de protección individual.

Palabras claves: Prevencíon \& Control; Apósitos oclusivos; Úlcera por presíon; Equipos y Suministros.

\section{Rafael Colodetti}

Doutorando do Programa de Pós-graduação em Biotecnologia da Universidade Federal do Espírito Santo (UFES). Mestre em Enfermagem (UFES). Enfermeiro Estomaterapeuta (UERJ). Fisioterapeuta (UVV). Especialista em Reabilitação Músculo-esquelética (EMESCAM).

ORCID: 0000-0001-7642-3702

\section{Walckiria Garcia Romero Sipolatti}

Doutora em Ciências Fisiológicas (UFES). Mestre em Ciências Fisiológicas (UFES). Professora do Curso de Mestrado Profissional em Enfermagem da UFES (PPGENF). Professora do Curso de Graduação em Enfermagem da UFES.

ORCID: 0000-0002-1365-4797

Recebido em: 11/03/2021

Aprovado em: 05/04/2021

\section{Maria Edla de Oliveira Bringuente}

Doutora em Enfermagem (UFRJ). Mestre em Educação (UFES). Professora do Curso de Mestrado Profissional em Enfermagem da UFES (PPGENF).

ORCID: 0000-0002-5151-5368

INTRODUÇÃO

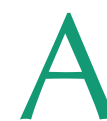
pandemia causada pelo agente etiológico do novo coronavírus SARS-CoV-2, conhecido como Covid-19, tem ocasionado grande ameaça a população mundial levando milhares de pessoas a procurar os serviços de saúde. Os primeiros relatos tiveram início em dezembro de 2019, quando uma série de casos de pneumonia de procedência desconhecida emergiu em
Wuhan, na China, com apresentações clínicas muito semelhantes à pneumonia viral ${ }^{(1)}$ com alta letalidade, principalmente, em pacientes imunocompetentes e com comorbidades associadas ${ }^{(2)}$.

Devido sua alta propagação entre pessoas, a Organização Mundial de Saúde (OMS) declarou em 30 de janeiro de 2020 o surto pela Covid-19, uma Emergência de Saúde Pública de interesse internacional e, em 11 de março do mesmo ano, foi caracterizada como uma pandemia ${ }^{(3)}$.

A forma de transmissão na população é principalmente entre pessoas após contato próximo e por meio de gotículas respiratórias, produzidas quando uma pessoa infectada tosse ou espirra. Profissionais de saúde são, particularmente, 
mais vulneráveis ao contágio, pois prestam assistência direta e próxima a esses pacientes. Em surtos anteriores provocados por outros tipos de coronavírus, profissionais de saúde representaram uma parcela expressiva no número de casos de contaminados, tendo contribuído para a ampliação das epidemias ${ }^{(4)}$.

$\mathrm{Na}$ tentativa de redução da contaminação pela Covid-19, o Ministério da Saúde orienta que todos profissionais de saúde, responsáveis pelo atendimento de casos suspeitos ou confirmados, utilizem equipamentos de proteção individual (EPI), tais como: gorro, óculos de proteção ou protetor facial, máscara, avental impermeável de mangas longas e luvas de procedimento $^{(4)}$. Dessa forma, com a utilização de medidas efetivas, a China conseguiu reduzir significativamente a transmissão pela Covid-19, sendo uma delas, a utilização rigorosa de EPI em todos os profissionais de saúde ${ }^{(5)}$.

Segundo a Agência Nacional de Vigilância Sanitária (ANVISA), os profissionais de saúde devem utilizar respirador de partícula N95 (máscara N95), peça facial filtrante-PFFP2 (máscara PFFP2), ou equivalentes, ao realizar procedimentos geradores de aerossóis como intubação ou aspiração orotraqueal, ventilação mecânica invasiva e não invasiva, ressuscitação cardiopulmonar, ventilação manual antes da intubação e coletas de amostras nasotraqueais. Tais máscaras garantem proteção em dois sentidos, porque tem um filtro de ar que bloqueia pelo menos 95\% das partículas em suspensão e ajuda na proteção contra doenças por transmissão aérea, como o coronavírus. Porém, a máscara deve estar apropriadamente ajustada à face para garantir sua eficácia e reduzir o risco de transmissão(6).

Apesar da orientação da OMS quanto ao uso de máscaras de proteção individual por até quatro horas ${ }^{(7)}$, durante a ocorrência da pandemia pela Covid-19, os EPI poderão ser utilizados por períodos superiores ao orientado pelo fabricante, devido à baixa disponibilidade desses materiais no ambiente de trabalho ${ }^{(6)}$
Dessa forma, a utilização prolongada e excessiva desse equipamento pode acarretar lesões por pressão em região da face dos profissionais de saúde, já claramente evidenciada na literatura ${ }^{(8-9)}$.

De acordo com a National Pressure Ulcer Advisory Panel (NPUAP) a lesão por pressão é um dano ou alteração na pele e tecidos moles, resultante da pressão contínua e prolongada sobre proeminências ósseas, também relacionada ao uso de dispositivos médicos, sendo recomendada a interrupção dessa força num período de até duas horas ${ }^{(10)}$.

É necessário ressaltar que a incidência e a prevalência das lesões por pressão, em pessoas sadias ou doentes, estão relacionadas com sensibilidade reduzida, restrição prolongada ao leito, idade avançada, doenças degenerativas, incontinência urinária ou intestinal, desnutrição ou obesidade $^{(11)}$.

No entanto, no decurso da pandemia, o surgimento de lesões por pressão na face dos profissionais de saúde, jovens e aparentemente saudáveis(8-9), traz evidências que o uso prolongado e rigoroso destes dispositivos de proteção são fatores importantes no desenvolvimento das mesmas.

\section{OBJETIVO}

Propor a elaboração de cobertura profilática para prevenção de lesão por pressão em áreas de risco da face, tais como narina e região zigomática, dos profissionais de saúde pelo uso de máscara durante a pandemia provocada pela Covid-19.

\section{MÉTODO}

\section{Tipo de estudo}

Trata-se de um estudo metodológico realizado nos meses de março e abril de 2020, pelo Programa de Pós-graduação Mestrado Profissional em Enfermagem da Universidade Federal do Espírito Santo, que desenvolve tecnologias de enfermagem para a assistência à população em geral. Para realização da pesquisa foram utilizadas duas etapas: 1 ) revisão narrativa de literatura e 2) proposta de elaboração da cobertura profilática.

Primeira etapa: revisão de literatura

A primeira etapa do estudo foi realizada por meio da revisão narrativa de literatura, onde os resultados deram subsídios para a escolha adequada das características e tipo do produto suscetíveis a compor a cobertura profilática. Para tal, foram acessadas as seguintes bases de dados: Medical Literature Analysis and Retrieval System Online (MEDLINE), Literatura Latino-Americana e do Caribe de Informação em Ciências da Saúde (LILACS). A busca das publicações da LILACS deu-se pela Biblioteca Virtual de Saúde (BVS) e os estudos da MEDLINE foram pesquisados por meio da BVS e do PubMed. Também foram acessados protocolos e guidelines do Ministério da Saúde e da OMS contendo informações sobre a Covid-19. Para nortear a pesquisa questionou-se quais tipos de curativos seriam mais adequados para compor uma cobertura profilática para prevenção de lesão por pressão relacionadas a uso de EPI.

Para tal, foram utilizados os Descritores em Ciência da Saúde (DeCS), em inglês e português "lesão por pressão", "prevenção e controle", "curativos oclusivos" e "equipamentos e provisões".

Segunda etapa: proposta de elaboração da cobertura profilática

Na segunda etapa da pesquisa, após revisão narrativa de literatura, os tipos de materiais que poderiam compor a cobertura profilática foram analisados pelos pesquisadores para posterior definição das características, assim como, dos gêneros mais adequados de produtos para serem utilizados na região da face. Logo, a composição dos insumos e características finais foram definidos pela revisão de literatura e pela experiência dos pesquisadores em estomaterapia e curativos especiais. 


\section{Critérios de seleção}

Para a seleção dos estudos encontrados na revisão narrativa de literatura, utilizou-se os seguintes critérios de inclusão: artigos originais, publicados no período de 2010-2020, em dois idiomas (inglês e português). Os critérios de exclusão foram: artigos duplicados, indisponíveis na íntegra e que não abordassem a temática.

Inicialmente foi realizada uma análise com base nos títulos e resumos de todos os artigos que preenchiam os critérios de inclusão. Após a leitura dos resumos foram obtidos na íntegra todos os artigos elegidos e, em seguida, examinados para confirmação de elegibilidade e inclusão no estudo.

Para a escolha dos produtos, os pesquisadores definiram como critérios de inclusão: componentes maleáveis, que se adequam anatomicamente à face, principalmente às regiões nasal e zigomáticas, capazes de absorver umidade, assim como, diminuir a pressão e o cisalhamento entre a pele e a máscara de proteção individual.

\section{Análise dos dados}

Para auxiliar na análise de dados, os estudos foram identificados e selecio- nados no item "Resultados" dos artigos, sendo digitados e organizados em uma planilha do programa Microsoft Office Excel® 2013 da seguinte forma: ano de publicação, autores, título do artigo e resultados. A análise de dados se deu de forma manual pelos pesquisadores.

\section{RESULTADOS}

Foram selecionados, na revisão narrativa de literatura, dez estudos científicos que auxiliaram os pesquisadores na escoIha dos produtos suscetíveis a compor a cobertura profilática (Quadro 1).

Quadro 1. Distribuição dos estudos científicos, encontrados na revisão de literatura, de acordo com o ano de publicação, autores, título e resultados, para a elaboração de cobertura profilática para prevenção de lesão por pressão na face dos profissionais de saúde durante a Covid-19. Vitória, ES, Brasil, 2020.

\begin{tabular}{|c|c|c|c|}
\hline $\begin{array}{l}\text { Ano de pu- } \\
\text { blicação }\end{array}$ & Autores & Título & Resultados \\
\hline 2018 & Moore ZEH, Webster J ${ }^{(12)}$ & $\begin{array}{l}\text { Dressings and topical agents for preventing pressure } \\
\text { ulcers. Cochrane Database of Systematic Reviews }\end{array}$ & $\begin{array}{c}\text { Embora a incidência de lesões por pressão } \\
\text { tenha sido reduzida quando os curativos foram } \\
\text { usados para proteger a pele, os resultados foram } \\
\text { comprometidos pela baixa qualidade dos estudos } \\
\text { incluídos. }\end{array}$ \\
\hline 2012 & Brindle CT, Wegelin JA ${ }^{(14)}$ & $\begin{array}{l}\text { Prophylactic dressing application to reduce pressure } \\
\text { ulcer formation in cardiac surgery patients }\end{array}$ & $\begin{array}{l}0 \text { grupo de paciente que recebeu tratamento } \\
\text { padrão teve uma taxa de risco 3,6 maior de } \\
\text { desenvolvimento de lesão por pressão, quando } \\
\text { comparado ao grupo que recebeu intervenção } \\
\text { com curativo com bordas de silicone, mas essa } \\
\text { diferença não foi estatisticamente significativa. }\end{array}$ \\
\hline 2012 & Chaiken $\mathrm{N}^{(16)}$ & $\begin{array}{l}\text { Reduction of sacral pressure ulcers in the intensive } \\
\text { care unit using a silicone border foam dressing }\end{array}$ & $\begin{array}{l}\text { O uso de curativo composto por espuma e com } \\
\text { borda de silicone conseguiu reduzir a taxas de } \\
\text { incidência de lesão por pressão de } 13,6 \% \text { para } \\
1,8 \% \text {. }\end{array}$ \\
\hline 2016 & Black J, Kalowes $\mathrm{P}^{(17)}$ & Medical device-related pressure ulcers & $\begin{array}{l}\text { O uso de curativos de espuma de silicone reduziu } \\
\text { a formação de lesões por pressão associadas a } \\
\text { dispositivo médico. }\end{array}$ \\
\hline
\end{tabular}




\begin{tabular}{|c|c|c|c|}
\hline 2016 & $\begin{array}{l}\text { Truong } B_{1} \text { Grigson } E_{1} \\
\text { Patel M, Liu X } X^{(19)}\end{array}$ & $\begin{array}{l}\text { Pressure ulcer prevention in the hospital setting } \\
\text { using silicone foam dressings }\end{array}$ & $\begin{array}{l}\text { Curativos de espuma de silicone, quando usados } \\
\text { como tratamento profilático, parecem ser superio- } \\
\text { res à conduta padrão de prevenção de lesões por } \\
\text { pressão. }\end{array}$ \\
\hline 2014 & $\begin{array}{c}\text { Clark M, Black J, Alves P, } \\
\text { Brindle CT, Call E, Dealey } \\
\text { C, Santamaria N(20) }\end{array}$ & $\begin{array}{l}\text { Systematic review of the use of prophylactic dressin- } \\
\text { gs in the prevention of pressure ulcers }\end{array}$ & $\begin{array}{l}\text { O uso de curativos tópicos reduzem a incidência } \\
\text { de lesões por pressão, assim como, das lesões } \\
\text { associadas a dispositivos médicos, em pacientes } \\
\text { em Unidade de Terapia Intensiva. }\end{array}$ \\
\hline 2015 & $\begin{array}{l}\text { Dutra RAA, Salomé GM, } \\
\text { Alves JR, Pereira VOS, } \\
\text { Miranda FD, Vallim VB, } \\
\text { Brito MJA, Ferreira LM }{ }^{(21)}\end{array}$ & $\begin{array}{l}\text { Using transparent polyurethane film and hydrocol- } \\
\text { loid dressings to prevent pressure ulcers }\end{array}$ & $\begin{array}{l}\text { O filme transparente de poliuretano teve melhor } \\
\text { desempenho e foi mais eficaz que o curativo } \\
\text { hidrocoloide na prevenção do desenvolvimento de } \\
\text { lesões por pressão. }\end{array}$ \\
\hline
\end{tabular}

A cobertura profilática proposta neste estudo teve origem a partir da análise crítica dos estudos científicos sobre os tipos de produtos que poderiam compor a cobertura profilática, além da experiência clínica dos pesquisadores sobre curativos especiais para prevenção das lesões por pressão.

Assim, a proposta é que a cobertura profilática tenha apresentação em rolo, não estéril, com cinco metros de comprimento. Consistirá em uma fita de silicone macio perfurado, formando uma camada de contato com micro-aderência, de 2,5 $\mathrm{cm}$ de largura e remoção atraumática, sendo utilizada somente em pele íntegra. Tal produto também permitirá a passagem de umidade, que será absorvida rapidamente pela camada de espuma extrafina de poliuretano com $1 \mathrm{~cm}$ de largura, localizada na porção central da fita, de excelente flexibilidade, além de capacidade para amenizar as forças de pressão e cisalhamento.

Figura 1. Proposta de cobertura profilática em rolo, não estéril, para redução das lesões por pressão na face dos profissionais de saúde pelo uso de máscaras de proteção individual durante a pandemia da Covid-19. Composta por camada de silicone perfurado (A e B), espuma de poliuretano (C) e filme de poliuretano (D).
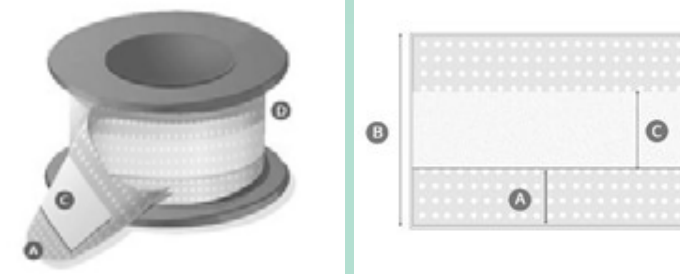
me de poliuretano com alta taxa de transmissão de vapor úmido, sendo inclusive, uma barreira contra à entrada de microrganismos. A apresentação em rolo possibilitará o corte e o uso conforme a necessidade do profissional de saúde. Dessa forma, a proposta da cobertura profilática apresenta-se no seguinte formato, conforme figura 1.

\section{DISCUSSÃO}

Este estudo propõe a elaboração de cobertura profilática, não estéril, para redução das lesões por pressão na face dos profissionais de saúde, por meio da redistribuição de pressão e absorção da umidade da pele sob a máscara de proteção individual utilizada por longos períodos, durante pandemia provocada pela Covid-19.

A prevenção destas lesões constitui uma ação emergencial, visto que, tal agravo causa dor e desconforto produzidos pela isquemia tecidual durante a pressão local ${ }^{(22)}$. Ademais, considerando a pele como um mecanismo de defesa à invasão de microrganismos, essas lesões podem comprometer a saúde dos profissionais, além de causar constrangimento, baixa autoestima, distúrbio da autoimagem, estigmatização e sentimentos de inferioridade.

O National Pressure Injury Advisory Panel (NPIAP) define que máscaras, óculos, bem como, quaisquer EPI são considerados dispositivos médicos e estes podem causar lesões de pele por meio da pressão e cisalhamento, além das alterações de microclima ${ }^{(23)}$. O microclima é a combinação de temperatura e umidade e, às vezes, do fluxo de ar em uma região local, em comparação com a área ambiente ou a área circundante, onde seu equilibrio é um fator importante na prevenção de lesões ${ }^{(24)}$.

A umidade é um fator causal primário no surgimento das lesões por pressão ao diminuir a permeabilidade da pele íntegra, enfraquecendo a epiderme e a derme que, consequentemente, se tornarão mais vulneráveis aos efeitos da pressão e do cisalhamento ${ }^{(25)}$. A umidade excessiva da pele leva a hidratação excessiva e danifica a função normal de barreira, aumentando o risco de lesão ${ }^{(26)}$.

Observamos, então, que a formação da lesão por pressão nos profissionais de saúde pelo uso de máscara não está ligada somente a pressão e cisalhamento. A utilização de máscaras de proteção 
individual por períodos superiores aos recomendados, associada ao estresse e excesso de carga de trabalho, podem promover alteração do microclima pela elevação da temperatura local e aumento da transpiração, contribuindo para a diminuição do movimento do ar que, consequentemente, promove o desenvolvimento das lesões ${ }^{(24)}$. Esses fatores de risco, em conjunto, causam deformação tecidual, edema inflamatório e isquemia que ocasionam as lesões por pressão em locais anatômicos ósseos imitando a forma e a distribuição dos dispositivos médicos utilizados na face ${ }^{(27)}$. Pesquisa recente demonstra que a região nasal foi a estrutura anatômica mais acometida pelas lesões por pressão causadas pelo uso de EPI, seguida pela região zigomática, sendo causadas principalmente pela transpiração da face ${ }^{(28)}$.

Estudo realizado por enfermeiras norte-americanas destaca três ações importantes na prevenção de lesões por pressão: considerar a aplicação de curativos que promovam a redistribuição de pressão e absorvam a umidade do corpo nas áreas em contato com dispositivos médicos e fixadores; aplicar curativos abaixo dos dispositivos médicos, levantar e/ou mover o dispositivo frequentemente para examinar a pele abaixo do mesmo; e reposicionar para alívio de pressão ${ }^{(29)}$.

Assim, o conhecimento de estratégias eficazes de prevenção representa uma poderosa ferramenta no gerenciamento do cuidado às pessoas sob risco de desenvolver lesões. É necessária a atuação de um profissional de enfermagem envolvido e com conhecimento científico, capaz de reconhecer os fatores de risco para o desenvolvimento de lesão por pressão, podendo desta maneira intervir de forma eficaz no uso dos materiais e coberturas mais adequados ${ }^{(30)}$.

Para fins de prevenção das lesões por pressão, os tipos de coberturas encontradas na literatura foram: curativos semipermeáveis (uma fina membrana de poliuretano revestida com uma camada de adesivo acrílico); hidrocoloides (cura- tivo contendo gelatina, pectina e carboximetilcelulose, juntamente com outros polímeros e adesivos formando um penso flexível); espuma (de poliuretano porosa, hidrofóbica); espuma multicamadas com silicone (curativo revestido com silicone como adesivo ou camada de contato com a ferida). Esses agentes tópicos podem ser usados isoladamente, sendo mais adequado, o uso em combinação ${ }^{(12)}$. Outros tipos de curativos, encontrados na revisão de literatura, não foram incluídos neste estudo pois não se enquadram à finalidade de prevenção e, sim, no tratamento de lesões por pressão.

As coberturas, descritas na revisão de literatura, foram avaliadas pelos pesquisadores e definiu-se que a proposta pelo produto em silicone deu-se ao fato deste já ter demonstrado eficácia na diminuição da umidade e prevenção no surgimento das lesões por pressão relacionadas ao uso de EPI ${ }^{(13)}$.

A eficácia de curativos e produtos tópicos na prevenção de lesões por pressão foram avaliados em nove estudos de uma revisão sistemática e os pesquisadores chegaram à conclusão de que os curativos de silicone podem reduzir a incidência de lesão por pressão, contudo como o nível de evidência ainda é baixo, são necessárias mais pesquisas para confirmar esse resultado ${ }^{(12)}$.

Porém, outros estudos clínicos descrevem a importância de curativos de espuma na prevenção das lesões por pressão e destacam diferenças significativas na incidência de lesões por pressão, na região sacral, em pacientes com uso de curativos de espuma de silicone macia com multicamadas, antes da transferência para a Unidade de Tratamento Intensivo $^{(15)}$, reforçando que a aplicação de cobertura profilática contendo silicone pode prevenir o surgimento de lesões por pressão. A incidência de lesão por pressão diminuiu de $13,6 \%$ para 1,8\% após a aplicação do curativo com bordas de silicone, indicando que esse tipo de cobertura previne efetivamente a incidência de lesão por pressão em região 
sacral de pacientes graves ${ }^{(16)}$. Da mesma forma, contribuiu com a redução de lesões por pressão associadas a dispositivo médico ${ }^{(17)}$.

Além do silicone, a proposta de combinação com a espuma extrafina de poliuretano deve-se ao fato de que esse produto também poderá agregar redução da pressão e maior absorção da umidade. As coberturas contendo espuma extrafina de poliuretano são absorventes e podem ser indicadas para tratamento e prevenção de lesões por pressão. Corroborando, dados de quatro estudos clínicos com 467 pacientes demostraram uma redução de $84 \%$ na incidência de lesões por pressão pelo uso de espuma de poliuretano quando comparado à placa de hidrocoloide ${ }^{(18)}$.

Os curativos de espuma de poliuretano ajudam a reduzir a pressão vertical, o cisalhamento e o atrito sobre o tecido em até $50 \%$, impedindo efetivamente a ocorrência das lesões por pressão ${ }^{(19)}$. Estudo, in vitro, demonstrou que a espuma de poliuretano, quando comparada às outras coberturas, é melhor na absorção, retenção e liberação de umidade, visto que, mantém o equilíbrio na preservação do microclima adequado evitando maceração da pele ${ }^{(14)}$. Além disso, a utilização de curativo de silicone macio ou filme de poliuretano, para prevenção de lesões por pressão sacrais, demonstrou ser mais eficaz em comparação à ausência de curativo local (20).

As espumas de poliuretano também minimizam a dor e o trauma durante a troca de curativo, por terem micro-aderência seletiva ${ }^{(28)}$ e a proposta de mudança da cobertura em períodos de até 12 horas poderá reduzir o risco de injúria da pele causada pelo atrito, já que desse modo, evitam-se remoções frequentes.

Outra proposta, é que a parte externa da cobertura profilática seja composta de filme transparente de poliuretano e resultados de um estudo sugerem que este produto teve melhores efeitos, sendo mais efetivo que os curativos de hidrocoloide na prevenção de lesões por pressão,
A cobertura profilática não estéril poderá contribuir com o aumento da adesão dos profissionais ao uso de EPI durante o período de pandemias, pelo baixo custo, praticidade e pela agradável sensação de alívio ao reduzir as pressões e lesões locais. principalmente por apresentarem alta capacidade de transmissão de vapor úmido, além de serem uma barreira contra a entrada de microrganismos ${ }^{(21)}$.

Da mesma forma, a maleabilidade da cobertura profilática permitirá ajuste ao formato anatômico da face e contribuirá para redução da pressão local. Além disso, a proposta de apresentação da cobertura profilática em rolo não estéril torna-se uma inovação, ao passo que os curativos existentes se limitam a proteção de pele somente de regiões de narinas e são comercialmente onerosos por apresentar, em sua grande maioria, placas individuais estéreis.

Deve-se considerar, ainda, que o uso de uma cobertura profilática sob a máscara, não substitui outras estratégias de prevenção, sendo fundamental a inspeção da pele, observando os sinais de pressão, devido ao uso do EPI e que o risco de lesões por pressão na face, causadas por estes dispositivos, não se restringe ao período da pandemia provocada pela Covid-19.

\section{Contribuiçõos para a enfermagem}

A cobertura profilática não estéril poderá contribuir com o aumento da adesão dos profissionais ao uso de EPI durante o período de pandemias, pelo baixo custo, praticidade e pela agradável sensação de alívio ao reduzir as pressões e lesões locais.

Limitação do estudo

Como limitação desse estudo destaca-se a baixa produção cientifica sobre o tema em questão, resultando em número reduzido de citações específicas que poderiam corroborar com as discussões realizadas neste estudo.

\section{CONCLUSÃO}

A cobertura profilática, proposta neste estudo, é composta por uma superfície de espuma de poliuretano extrafina, maleável e absorvente, em combinação, com uma camada de contato de silicone transparente perfurada para auxiliar a fi- 
xação na pele. Assim, reduz a umidade, a pressão e o cisalhamento da pele, en- quanto permite que a máscara, independente do modelo ou variações anatômi- cas individuais, atenda sua finalidade de proteção.

\section{Referências}

1. World Health Organization. Novel coronavirus - China. World Health Organization [Internet]. 2020 (cited 2020 Mar 29). Available from: http://www.who. int/csr/don/12-january-2020-novel-coronavirus-china/en/

2. Benvenuto D, Giovanetti M, Ciccozzi A, Spoto S, Angeletti S, Ciccozzi M. The 2019-new coronavirus epidemic: Evidence for virus evolution. J Med Virol. 2020; 1-5. DOI: 10.1002/jmv.25688

3. Organização Pan-Americana da Saúde (OPAS). OMS afirma que COVID-19 é agora caracterizada como pandemia [Internet]. 2020 (cited 2020 Mar 29). Available from: https://www.paho.org/bra/index.php?option=com content\&view=article\&id=6120:oms-afirma-que-covid-19-e-agora-caracterizada-como-pandemia\&ltemid $=812$

4. Brasil. Ministério da Saúde. Protocolo de Tratamento do Novo Coronavírus (2019-nCoV) (Internet). Brasília; 2020 (cited 2020 Apr 04). Available from: https://www.arca.fiocruz.br/bitstream/icict/40195/2/Protocolo_Tratamento_Covid19.pdf

5. World Health Organization. Report of the WHO-China Joint Mission on Coronavirus Disease 2019 (COVID-19). World Health Organization [Internet]. 2020 (cited 2020 Mar 06). Available from: https://www.who.int/docs/default-source/ coronaviruse/who-china-joint-mission-on-Covid-19-final-report.pdf 6. Brasil. Nota técnica GVIMS/GGTES/ANVISA $n^{\circ}$ 04/2020. Orientações para serviços de saúde: medidas de prevenção e controle que devem ser adotadas durante a assistência aos casos suspeitos ou confirmados de infecção pelo novo coronavírus (SARS-CoV-2) [Internet]. (Atualizada em 21/03/2020). 2020 (cited 2020 Apr 01). Available from: http:/l portal.anvisa.gov.br/documents/33852/271858/Nota+T\%C3\%A9cni$\mathrm{ca}+\mathrm{n}+04-2020+$ GVIMS-GGTES-ANVISA/ab598660-3de4-4f14-8e6fb9341c196b28.

7. OPAS/OMS Brasil - Organização Pan-Americana da Saúde. Máscaras faciais durante surtos: quem, quando, onde e como usá-las [Internet]. 2020. (cited 2020 Mar 29). Available from: https://www.paho.org/bra/index.php?option $=$ com_content\&view=article\&id=6115: mascarasfaciais-durante-surtos-quem-quando-onde-e-como-usa-las\&ltemid $=812$.

8. Utaraite N. Chinese nurses share pictures of how their faces look after countless hours fighting the coronavirus. Bored Panda [Internet]. 2020 (cited 2020 Mar 30). Available from: https://www.boredpanda.com/chinese-nurses-face-masks-coronavirus $/$ ?utm_source=google\&utm_medium =organic\&utm_campaign=Organic

9. Ramalho AO, Freitas PSS, Nogueira PC. Lesão por pressão relacionada a dispositivo médico nos profissionais de saúde em época de pandemia. Estima. Braz. J. Enterostomal Ther. São Paulo. 2020; 18: e0120. DOI: 10.30886/ estima. v18.867_PT

10. National Pressure Ulcer Advisory Panel (NPUAP). National Pressure Ulcer Advisory Panel (NPUAP) announces a change in terminology from pressure ulcer to pressure injury and updates the stages of pressure injury [Internet] 2016 (cited 2020 Mar 29). Available from: http://www.npuap.org/national-pressure-ulcer-advisory-panel-npuap-announces-a-change-in-terminology-from-pressureulcer-to-pressure-injury-and-updates-the-stages-of-pressure-injuryl

11. Palagi S, Severo IM, Mengon DB, Lucena AF. Laser therapy in pressure ulcers: evaluation by the Pressure Ulcer Scale for Healing and Nursing Outcomes Classification. Rev Esc Enferm USP. São Paulo. 2015; 49 (5): 820-826. DOI: 10.1590/S0080-623420150000500017

12. Moore_ZEH, Webster_J. Dressings and topical agents for preventing pressure ulcers. Cochrane Database of Systematic Reviews. 2018; 12. DOI: 10.1002/14651858.CD009362.pub3

13. Burch J, Tort S. Can silicone dressing help prevent pressure ulcers? Cochrane Clinical Answers. 2019. DOI: 10.1002/cca.2386

14. Brindle CT, Wegelin JA. Prophylactic dressing application to reduce pressure ulcer formation in cardiac surgery patients. J Wound Ostomy Continence
Nurs. 2012; 39: 133-142. DOI: 10.1097/WON.0b013e318247cb82

15. Santamaria N, Gerdtz M, Sage S, McCann J, Freeman A, Vassiliou T, et al. A randomized controlled trial of the effectiveness of soft silicone multi-layered foam dressings in the prevention of sacral and heel pressure ulcers in trauma and critically ill patients: the border trial. Int. Wound J. 2013; 12 (3): 302-308. DOI: 10.1111/iwj.12101

16. Chaiken N. Reduction of sacral pressure ulcers in the intensive care unit using a silicone border foam dressing. J. Wound Ostomy Continence Nurs. 2012; 39, 143-145. DOI: 10.1097/WON.0b013e318246400c

17. Black J, Kalowes P. Medical device-related pressure ulcers. Chronic Wound Care Management and Research. 2016; 3: 91-99. DOI: 10.2147/CWCMR. S82370

18. Huang L, Woo K, Liu L, Wen R, Hu A, Shi C. Dressings for preventing pressure ulcers: a meta-analysis. Advances in Skin \& Wound Care. 2015; 28 (6): 267-273 7p. DOI: 10.1097/01.ASW.0000463905.69998.0d

19. Truong B, Grigson E, Patel M, Liu X. Pressure ulcer prevention in the hospital setting using silicone foam dressings. Cureus. 2016; 8: e730. DOI: 10.7759/ cureus. 730

20. Clark M, Black J, Alves P, Brindle CT, Call E, Dealey C, Santamaria N. Systematic review of the use of prophylactic dressings in the prevention of pressure ulcers. Int J ferida. 2014; 11 (5): 460-471. DOI: 10.1111/iwj.12212

21. Dutra RAA, Salomé GM, Alves JR, Pereira VOS, Miranda FD, Vallim VB, Brito MJA, Ferreira LM. Using transparent polyurethane film and hydrocolloid dressings to prevent pressure ulcers. Journal of Wound Care. 2015; 24 (60), 268-327. DOI: 10.12968/jowc.2015.24.6.268

22. Otto C, Schumacher B, Wiese LPL, Ferro C, Rodrigues RA. Fatores de risco para o desenvolvimento de lesão por pressão em pacientes críticos. Enferm Foco. 2019; 10 (1): 07-11. DOI: 10.21675/2357-707X.2019.v10.n1.1323

23. European Pressure Ulcer Advisory Panel (EPUAP), National Pressure Ulcer Advisory Panel (NPUAP), Pan Pacic Pressure Injury Alliance (PPPIA). Prevention and treatment of pressure ulcers: quick reference guide. Haesler $\mathrm{E}(\mathrm{ed})$ [Internet]. 2014 (cited 2020 Apr 07). Available from: https://www.epuap. org/wp-content/uploads/2010/10/Quick-Reference-Guide-DIGITAL-NPUAPEPUAP-PPPIA-160ct2014.pdf

24. Kottner J, Black J, Call E, Gefen A, Santamaria N. Microclimate: a critical review in the context of pressure ulcer prevention. Clin Biomech. 2018; 59: 62-70. DOI: 10.1016/j.clinbiomech.2018.09.010

25. Woo KY, Beeckman D, Chakravarthy D. Management of moisture-associated skin damage: a scoping review. Adv Skin Wound Care. 2017; 30 (11): 494-501. DOI: 10.1097\%2F01.ASW.0000525627.54569.da

26. Demarre L, Verhaeghe $S$, Van Hecke A, Clays E, Grypdonck M, Beeckman D. Factors predicting the development of pressure ulcers in an at-risk population who receive standardized preventive care: secondary analyses of a multicentre randomised controlled trial. J Adv Nurs. 2015; 71: 391-403. DOI: 10.1111/ jan.12497

27. Gefen A, Alves P, Ciprandi G, Cover F, Milne CT, Ousey K, Ohura N, Waters N, Worsley P. Device related pressure ulcers: SECURE prevention. J Wound Care. 2020; 29 (Sup2a): S1-S52. DOI: 10.12968/jowc.2020.29.Sup2a.S1

28. Otero DP, Domínguez DV, Fernández LH, Magariño AS, González VJ, Klepzing JVG, Montesinos JVB. Preventing facial pressure ulcers in patients under non-invasive mechanical ventilation: a randomised control trial. Journal of Wound Care. 2017; 26 (3), 128-136. DOI: 10.12968/jowc.2017.26.3.128

29. Black JM, Alves P, Brindle CT, Dealey C, Santamaria N, Call E, et al. Use of wound dressings to enhance prevention of pressure ulcers caused by medical devices. Int Wound J. 2015; 12 (3): 322-327. DOI: 10.1111/iwj.12111

30. Araujo M, Castanheira L, Guimarães MC, Silva Y. Análise de custo da prevenção e do tratamento de lesão por pressão: revisão sistemática. Revista Enfermagem Atual [Internet]. 2019; 89 (27). Available from: http://www.revistaenfermagematual.com.br/index.php/revista/article/view/47 